\title{
Erratum to: The Politics, Practices, and Possibilities of Migrant Children Schools in Contemporary China
}

\author{
$\operatorname{Min} \Upsilon u$
}

DOI 10.1057/978-1-137-50900-0

Erratum to: Notes, Glossary pages and References The errors of the Chinese characters in note 9 on page 130 and in the glossary pages $177-180$ are corrected.

The references on pages 181-185 are deleted.

The reference list on pages 185-186 are moved to page 170, the end of Appendix A.

"References" are deleted from the ToC and page numbers updated accordingly.

The online version of the original book can be found under DOI 10.1057/978-1-137-50900-0 